\title{
Estudo SABE: Fatores associados ao uso de medicamentos para controle da dor crônica em idosos
}

\author{
SABE study: Factors associated with the use of drugs to control chronic pain \\ in the elderly
}

Eduardo Godoi Audi (D) 1, Mara Solange Gomes Dellaroza (D) 1, Marcos Aparecido Sarria Cabrera (D) 2, Hellen Geremias dos Santos (D) 3, Camila Helen de Oliveira Bettiol (D) 1, Dayane Aparecida Scaramal (D) $4 \bowtie$

' Universidade Estadual de Londrina, Departamento de Enfermagem. Londrina, Paraná, Brasil.

2 Universidade Estadual de Londrina, Departamento de Saúde Coletiva. Londrina, Paraná, Brasil.

${ }^{3}$ Fundação Oswaldo Cruz, Instituto Carlos Chagas. Curitiba, Paraná, Brasil.

${ }^{4}$ Universidade Pitágoras - Unopar, Departamento de Enfermagem. Londrina, Paraná, Brasil.

Como citar este artigo (How to cite this article):

Audi EG, Dellaroza MSG, Cabrera MAS, Santos HG, Bettiol CHO, Scaramal DA. Estudo SABE: Fatores associados ao uso de medicamentos para controle da dor crônica em idosos (SABE study: Factors associated with the use of drugs to control chronic pain in the elderly). Sci Med. 2019;29(4):e34235. https://doi.org/10.15448/1980-6108.2019.4.34235

\section{RESUMO}

OBJETIVO: Analisar os fatores associados ao uso de medicamentos para controle da dor crônica por idosos do município de São Paulo. MÉTODOS: Estudo transversal com utilização de dados do Estudo Saúde, Bem-Estar e Envelhecimento (SABE) que teve início no ano 2000, com reentrevistas em 2006 e 2010. Para esta pesquisa, inicialmente, foram utilizados dados dos idosos que foram reentrevistados em 2010. Dessa amostra, os idosos que apresentaram dor crônica foram selecionados para a análise de fatores associados ao uso de medicamentos para controle da dor. Para a identificação desses fatores utilizou-se o teste de Rao Scott. Por se tratar de estudo com desenho amostral complexo, para todas as análises considerou-se pesos amostrais estimados para o seguimento de 2010.

RESULTADOS: Da amostra inicial de 978 idosos, 303 (30,98\%) relataram dor crônica. Entre esses idosos, observou-se menor frequência de utilização de analgésicos para os que referiram ter realizado a última consulta médica em convênio/particular $(\mathrm{OR}=0,55$; IC $95 \%$ : 0,31-0,96); menor frequência de utilização de antidepressivos para os que referiram não ter plano de saúde $(\mathrm{OR}=0,49$; IC 95\%: 0,24-0,98); e maior utilização de sintomáticos para dispepsia para os com autopercepção de saúde regular $(\mathrm{OR}=2,20 ; \mathrm{IC} 95 \%$ : 1,12-4,32) e para os que referiram sentir dor diariamente $(\mathrm{OR}=2,24$; IC 95\%: 1,31-3,81).

CONCLUSÕES: Dessa maneira conclui-se que a dor é um fator que afeta diretamente a vida do idoso e, estes, muitas vezes, buscam o alívio para seu sofrimento nos medicamentos, e o fato de realizar consultas médicas em serviço privado ou conveniado aumentam a frequência de utilização de determinados medicamentos.

DESCRITORES: Dor crônica, idoso, epidemiologia, uso de medicamentos, serviços de saúde.

\section{ABSTRACT}

AIMS: This study aims to analyze the factors associated with the use of medications to control chronic pain in the elderly, study carried out in the city of São Paulo.

METHODS: This a cross-sectional study using data from the Health, Welfare and Ageing Study (SABE), which began in 2000 with reinterviews in 2006 and 2010. For this research, data from elderly people re-interviewed in 2010 were initially used. From this sample, elderly individuals showed chronic pain were selected for the analysis of factors associated with the use of medications for pain control. The Rao Scott test was used to identify these factors. Since this is a study with a complex sample design, estimated sample weights for the 2010 follow-up were considered for all analyses.

RESULTS: From the initial sample of 978 elderly people, 303 (30.98\%) reported chronic pain. Among these elderly people, there was a lower frequency of using analgesics for those who reported having made the last medical consultation in a health care $/$ private $(\mathrm{OR}=0,55$; IC $95 \%$ : 0,31-0,96); lower frequency of using antidepressants for those who reported not having health insurance $(\mathrm{OR}=0,49$; IC $95 \%$ : $0,24-0,98)$; greater use of symptomatic for dyspepsia; for those with regular health self-perception (OR=2,20; IC 95\%: 1,12-4,32); and for those who reported feeling pain daily $(\mathrm{OR}=2,24$; IC $95 \%: 1,31-3,81)$

CONCLUSIONS: Thus, it is concluded that pain is a factor that directly affects the life of the elderly and they often seek relief for their suffering in medications, and the fact of performing medical consultations in a private care or health service increases the frequency of use of certain medications.

KEYWORDS: Chronic pain, aged, epidemiology, drug utilization, health services. 
Abreviaturas: IC, intervalo de confiança; OR, Odds Ratio; SABE, Saúde, Bem-Estar e Envelhecimento; SUS, Sistema Único de Saúde.

\section{INTRODUÇÃO}

A população mundial está envelhecendo, enquanto que a taxa global de fertilidade nos últimos 29 anos passou de 3,2 nascimentos por mulher para 2,5. Este cenário foi evidente no ano de 2018, uma vez que a quantidade de idosos ultrapassou o número de crianças menores de cinco anos no mundo. Para 2050, estima-se que uma em cada seis pessoas no mundo terá mais de 65 anos, representando $16 \%$ da população mundial, enquanto que em 2019 o valor é de $9 \%$. A perspectiva para os próximos 30 anos é que a população com 65 anos ou mais deve dobrar em países do norte da África, Ásia, América Latina e Caribe [1].

No Brasil o número de idosos cresceu significativamente entre 2005 e 2015 . A proporção de idosos com 60 anos ou mais passou de $9,8 \%$ para $14,3 \%$ neste período, enquanto a proporção de crianças de 0 a 14 anos, caiu em 5,5 pontos percentuais, e a de jovens de 15 a 19 anos, caiu 3,8, evidenciando envelhecimento demográfico [2]. Tais transformações implicam na elevação de custos para o Estado em detrimento da demanda aumentada por saúde. Neste sentido, é preciso que a sociedade e o Estado compreendam que o envelhecimento biológico provoca alterações anatômicas e fisiológicas, evidenciadas pelo aparecimento de sintomas, como a dor.

A dor crônica é a principal queixa e causa de muitas limitações funcionais em idosos, e está diretamente relacionada à piora da qualidade de vida, devido elevada frequência e intensidade [3]. A Associação Internacional de Estudo da Dor determina que a diferença temporal entre dor aguda e crônica é de três meses. Mais precisamente dor crônica é aquela sentida em uma ou mais regiões anatômicas, contínua ou recorrente de duração mínima de três meses, além de estar associada ao sofrimento emocional relevante e/ou incapacidade funcional significativa [4].

Os idosos frequentemente relatam apresentar episódios diários de dor intensa que pode se prolongar por mais de dois anos, comprometendo sua mobilidade e interferindo negativamente na capacidade para as atividades diárias básicas e instrumentais de vida [5]. Entretanto a dor apresenta-se como um dos problemas mais relevantes que dificultam consideravelmente a autonomia do idoso no desempenho de suas funções.
Além disso, é uma das principais causas de polifarmácia e iatrogenia terapêutica [6].

O uso de medicamentos para o controle da dor crônica expõe o idoso a complicações em decorrência das alterações relacionadas à metabolização desses fármacos, oferecendo maior possibilidade de eventos adversos e iatrogenia. Desse modo, muitas vezes, tornase necessário o uso de medidas não farmacológicas associadas ao tratamento medicamentoso, possibilitando o uso de menor número de drogas e menores doses, reduzindo assim, o risco de efeitos indesejáveis [7].

Em relação aos analgésicos, estes podem ser divididos em opioides e não opioides. Os opioides são utilizados para tratamento da dor moderada ou intensa, seja aguda ou crônica, presente em pacientes com câncer ou outras síndromes dolorosas graves. Os medicamentos mais utilizados são codeína, propoxifeno, tramadol, morfina e metadona, que apresentam como efeitos colaterais náuseas, vômitos, tonturas, obstipação, deterioração cognitiva, depressão respiratória e mioclonias [8].

Já os analgésicos não opioides, também classificados como drogas anti-inflamatórias não hormonais, são frequentemente usados em casos de dores esporádicas e leves e os mais comuns são a dipirona, o ácido acetilsalicílico e o paracetamol. Também podem apresentar efeitos colaterais, pois o ácido acetilsalicílico está associado ao aparecimento de gastrite e hemorragia gastrointestinal, a dipirona pode acarretar hipotensão postural e fenômenos alérgicos e o paracetamol pode induzir insuficiência hepática aguda [8]. Considerando a elevada probabilidade de sangramento gastrointestinal em idosos e o potencial nefrotóxico, de portadores de lesão renal preexistente, estes medicamentos são contraindicados quando o uso for por tempo prolongado [8]. Desta forma, esta classe de medicamentos pode ser empregada no tratamento de dores musculoesqueléticas agudas e, principalmente, quando há sinais de artropatia inflamatória ou metástase óssea [8].

Em relação a este contexto, faz-se necessário destacar que a vulnerabilidade dos idosos aos eventos adversos relacionados aos medicamentos é alta, o que se deve a polimorbidade, associada à complexidade dos problemas clínicos e ao uso de várias drogas. Para tanto, a promoção do uso racional de medicamentos deve ser incentivada [9].

Visto que muitas vezes os idosos buscam o controle da dor por meio de medicamentos, pouco se conhece quais são os fatores que influenciam o uso. Sem informações sobre este tema não é possível mensurar 
o quanto a dor impacta no uso geral de medicamentos e os riscos que esta prática provoca. Dessa maneira o objetivo desse estudo foi analisar os fatores relacionados ao uso de medicamentos para controle da dor crônica por idosos residentes na comunidade.

A partir deste contexto a questão que norteou esta pesquisa foi: Quais fatores estão relacionados ao uso de medicamentos para o controle da dor crônica por idosos residentes na comunidade?

\section{MÉTODO}

Tratou-se de um estudo transversal, com utilização dos dados do Estudo Saúde, Bem-Estar e Envelhecimento (SABE) correspondentes ao seguimento realizado no ano 2010. O Estudo SABE teve início no ano 2000, financiado pela Organização PanAmericana de Saúde como um inquérito multicêntrico em sete centros urbanos na América Latina e Caribe, sendo eles: Bridgetown (Barbado); Buenos Aires (Argentina); São Paulo (Brasil); Santiago (Chile); Havana (Cuba); Cidade do México (México) e Montevidéu (Uruguai) [10].

Nos anos 2006, 2010 e 2015 o estudo teve continuidade com novas coletas somente no Brasil, e hoje, caracteriza-se por um estudo longitudinal de idosos da região Metropolitana do Município de São Paulo [11].

Para esta pesquisa, inicialmente, foram utilizados dados de idosos com idade igual ou superior a 60 anos, residentes no município de São Paulo, reentrevistados durante o seguimento de 2010. Dessa amostra, os idosos que apresentaram dor crônica foram selecionados para a análise de fatores associados ao uso de medicamentos para controle da dor.

Neste estudo, as seguintes variáveis foram analisadas: dor crônica, características socioeconômicas (escolaridade e se a renda é suficiente para seu sustento) e demográficas (sexo, idade e estado civil), doenças autorreferidas (hipertensão, diabetes, doença articular, doença cardíaca e osteoporose), se o idoso mora sozinho ou acompanhado, uso e acesso aos serviços de saúde (se tem plano de saúde, quando realizou a última consulta médica e financiamento da última consulta), autopercepção de saúde, presença de dor crônica e características da dor que mais incomoda (duração, local, frequência e intensidade) e uso de medicamentos para o controle da dor. Esses medicamentos foram agrupados de acordo com o terceiro nível da classificação internacional de medicamentos da Anatomical Therapeutic Chemical [12].

Os dados foram analisados com auxílio do IBM SPSS Statistics 20. Realizou-se análise exploratória dos dados por meio de distribuição de frequências de características socioeconômicas e demográficas, do estado de saúde (doenças autorreferidas) e do uso e acesso aos serviços de saúde, bem como variáveis referentes ao uso de medicamentos (número de medicamentos utilizados, gasto com medicamentos, como o idoso adquiriu o medicamento) e as características da dor que mais o incomoda (tempo, local, intensidade e frequência dos episódios de dor).

Foram realizadas análises bivariadas entre dor e o uso de medicações, utilizou-se como medida de associação o Odds Ratio (OR) com seus respectivos intervalos de confiança (IC) e para todas as variáveis independentes foi estabelecida uma categoria de referência $(\mathrm{OR}=1)$, considerada a de menor risco para a utilização da respectiva classe de medicamentos analisada como desfecho. O nível de significância adotado foi de 5\% (teste de Rao-Scott). Por se tratar de estudo com desenho amostral complexo, para todas as análises considerou-se pesos amostrais estimados para o seguimento de 2010.

O uso dos dados do Estudo SABE 2010: Saúde, Bem-Estar e Envelhecimento - Estudo longitudinal sobre as condições de vida e saúde dos idosos no município de São Paulo foi autorizado por sua coordenadora. $\mathrm{O}$ presente estudo foi submetido e aprovado pelo ao Comitê de Ética da Universidade Estadual de Londrina, conforme parecer número 1.863.153/2017.

\section{RESULTADOS}

Entre os 978 idosos reentrevistados no seguimento de 2010, 303 (30,98\%) relataram dor crônica. Quanto às características socioeconômicas e demográficas, o número maior de participantes foi do sexo feminino $632(61,4 \%)$; em relação a idade, a maior frequência, foi de 70 a 79 anos 384 (44,6\%); quanto ao estado civil, os idosos com companheiro representaram o maior número 449 (52,0\%); sobre a escolaridade, 780 $(79,8 \%)$ apresentaram escolaridade baixa, menor do que sete anos; a maior parte dos idosos $798(83,7 \%)$ referiu morar com outras pessoas e mais da metade $565(56,6 \%)$ considerou suficiente a renda para seu sustento.

As morbidades mais relatadas foram: hipertensão 695 (71,1\%), doença articular 348 (35,6\%), doença cardíaca $258(26,4 \%)$, diabetes $245(25,1 \%)$ e osteoporose $222(22,7 \%)$.

Quanto às características do uso e acesso aos serviços de saúde, 512 (53,2\%) não possuíam plano de saúde e $246(24,4 \%)$ relataram dificuldade para acessar/ utilizar serviços de saúde. Ao responderem quando foi 
a última consulta médica, a maior porcentagem dos idosos $792(81,0 \%)$ foi consultada no mínimo há seis, e observou-se maior frequência de consultas financiadas pelo Sistema Único de Saúde (SUS). Em relação à autopercepção de saúde, $856(87,6 \%)$ responderam perceber sua saúde como muito boa/boa ou regular.

$\mathrm{Na}$ amostra geral $(\mathrm{n}=978)$, entre as medicações usadas para o controle da dor mais citadas foram os analgésicos 262 (26,7\%), seguido dos antiácidos, medicamentos para tratamento da úlcera péptica e da flatulência 228 (22,3\%), psicoanalépticos $124(12,0 \%)$, anti-inflamatórios e antirreumáticos 108 (10,8\%) e psicolépticos $101(9,4 \%)$.

A maioria dos medicamentos foi obtida de forma gratuita, com porcentagem variando entre $45,4 \%$ (drogas antiácidas; medicamentos para tratamento da úlcera péptica e da flatulência) e 94,2\% (drogas gastrointestinais, agentes antiespasmódicos, anticolinérgicos e propulsivos).

Os medicamentos indicados para o controle de dor como analgésicos ou coadjuvantes, foram agrupados de acordo com o terceiro nível da classifica- ção internacional de medicamentos da Anatomical Therapeutic Chemical [12]. Entre todos os idosos entrevistados $(n=978)$, as seguintes frequências de utilização desses medicamentos foram observadas: analgésicos e antipiréticos 250 (25,6\%); antiinflamatórios e antirreumáticos não esteroides 105 (10,8\%); antidepressivos 104 (10,7\%); ansiolíticos 70 (7,2\%); antipsicóticos 19 (1,9\%); opioides $16(1,6 \%)$ e hipnóticos e sedativos $6(0,6 \%)$.

Entre os 303 idosos com dor crônica, as seguintes características foram observadas para a dor que mais incomodava: dor presente há mais de 24 meses 187 $(62,3 \%)$, localizada na pelve/pernas $103(10,5 \%)$, cabeça $68(7,0 \%)$ ou ombro/braço $55(5,9 \%)$, de intensidade média/moderada $122(40,0 \%)$ e de frequência diária $146(46,0 \%)$.

A Tabela 1 apresenta a relação entre medicamentos sintomáticos da dor (medicamento analgésicos, antirreumáticos, anti-inflamatórios ou relaxantes musculares) e características de uso e acesso aos serviços de saúde; autopercepção de saúde e características da dor que mais incomoda.

Tabela 1. Relação entre medicamentos sintomáticos da dor e características de uso e acesso aos serviços de saúde; autopercepção de saúde e características da dor que mais incomoda entre idosos com dor crônica $(n=303)$ - Estudo SABE, 2010, São Paulo.

\begin{tabular}{|c|c|c|c|c|c|}
\hline Variáveis & Total* & $\mathbf{n}$ & $\%$ & OR não ajustado (IC95\%) & $\mathbf{p}$ \\
\hline \multicolumn{6}{|l|}{ Plano de saúde } \\
\hline Sim & 118 & 48 & 40,2 & 1,00 & \multirow[t]{2}{*}{0,27} \\
\hline Não & 185 & 89 & 65,4 & $1,31(0,79-2,18)$ & \\
\hline \multicolumn{6}{|c|}{ Dificuldade para acessar/utilizar serviços de saúde } \\
\hline Sim & 112 & 53 & 45,9 & $1,08(0,65-1,79)$ & \multirow[t]{2}{*}{0,73} \\
\hline Não & 190 & 84 & 43,8 & 1,00 & \\
\hline \multicolumn{6}{|c|}{ Financiamento da última consulta médica } \\
\hline SUS & 164 & 81 & 66,2 & 1,00 & \multirow[t]{2}{*}{0,21} \\
\hline Convênio/Particular & 107 & 45 & 40,5 & $0,72(0,43-1,20)$ & \\
\hline \multicolumn{6}{|l|}{ Percepção de saúde } \\
\hline Muito boa/boa & 79 & 31 & 37,0 & 1,00 & \multirow[t]{3}{*}{0,16} \\
\hline Regular & 172 & 80 & 46,2 & $1,46(0,86-2,48)$ & \\
\hline Ruim/muito ruim & 40 & 22 & 54,2 & $2,01(0,91-4,44)$ & \\
\hline \multicolumn{6}{|l|}{ Dor $>24$ meses } \\
\hline Sim & 187 & 89 & 46,5 & $1,17(0,66-2,08)$ & \multirow[t]{2}{*}{0,58} \\
\hline Não & 113 & 48 & 42,5 & 1,00 & \\
\hline \multicolumn{6}{|l|}{ Dor pelve/pernas } \\
\hline Sim & 103 & 52 & 49,5 & $1,35(0,86-2,13)$ & \multirow[t]{2}{*}{0,17} \\
\hline Não & 200 & 85 & 41,9 & 1,00 & \\
\hline \multicolumn{6}{|c|}{ Dor moderada/forte/intensa } \\
\hline Sim & 239 & 118 & 47,7 & $1,69(0,96-2,96)$ & \multirow[t]{2}{*}{0,06} \\
\hline Não & 54 & 18 & 35,0 & 1,00 & \\
\hline \multicolumn{6}{|l|}{ Dor todos os dias } \\
\hline Sim & 146 & 73 & 50,0 & $1,57(0,98-2,50)$ & \multirow[t]{2}{*}{0,05} \\
\hline Não & 153 & 62 & 38,9 & 1,00 & \\
\hline
\end{tabular}

* Calculado com base nos pesos amostrais decorrentes do desenho amostral complexo. OR, Odds Ratio. 
A prescrição de medicamentos sintomáticos da dor não apresentou associação com características de uso e acesso aos serviços de saúde, autopercepção de saúde e características da dor. No entanto, chama a atenção o fato de que somente $239(47,7 \%)$ dos idosos com dor moderada a forte referiram utilizar medicamentos sintomáticos para dor.

Em relação aos medicamentos analgésicos, observou-se menor frequência de sua utilização entre idosos que referiram ter realizado a última consulta médica em convênio/particular $(\mathrm{OR}=0,55$; IC 95\%: 0,31-0,96), comparado àqueles que realizaram a consulta por financiamento do SUS (Tabela 2).

A Tabela 3 evidencia que o uso de medicamentos antirreumáticos e anti-inflamatórios por idosos com dor crônica não apresentou associação com características de uso e acesso aos serviços de saúde, autopercepção de saúde e características da dor que mais incomoda.

Em relação aos medicamentos antidepressivos, observou-se menor frequência de utilização entre idosos que referiram não ter plano de saúde $(\mathrm{OR}=0,49$; IC 95\%: 0,24-0,98) (Tabela 4).

Por fim, o uso de medicamentos sintomáticos para dispepsia apresentou associação com a autopercepção de saúde regular (OR=2,20; IC 95\%: 1,12-4,32), quando comparado aos idosos com autopercepção de saúde muito boa/boa, e que relataram frequência diária para a dor que mais incomoda $(\mathrm{OR}=2,24$; IC95\%: 1,31-3,81), identificados na Tabela 5.

Tabela 2. Relação entre medicamentos analgésicos e características de uso e acesso aos serviços de saúde, autopercepção de saúde e características da dor que mais incomoda entre os idosos com dor crônica $(n=303)$ - Estudo SABE, 2010, São Paulo.

\begin{tabular}{|c|c|c|c|c|c|}
\hline Variáveis & Total* & $\mathbf{n}$ & $\%$ & OR não ajustado (IC95\%) & $\mathbf{p}$ \\
\hline \multicolumn{6}{|l|}{ Plano de saúde } \\
\hline Sim & 118 & 35 & 28,9 & 1,00 & \multirow[t]{2}{*}{0,22} \\
\hline Não & 185 & 69 & 36,5 & $1,41(0,80-2,46)$ & \\
\hline \multicolumn{6}{|c|}{ Dificuldade para acessar/utilizar serviços de saúde } \\
\hline Sim & 112 & 42 & 36,4 & $1,21(0,75-1,94)$ & \multirow[t]{2}{*}{0,42} \\
\hline Não & 190 & 62 & 32,1 & 1,00 & \\
\hline \multicolumn{6}{|c|}{ Financiamento da última consulta médica } \\
\hline SUS & 164 & 66 & 40,0 & 1,00 & \multirow[t]{2}{*}{0,03} \\
\hline Convênio/Particular & 107 & 31 & 26,8 & $0,55(0,31-0,96)$ & \\
\hline \multicolumn{6}{|l|}{ Percepção de saúde } \\
\hline Muito boa/boa & 79 & 23 & 22,8 & 1,00 & \multirow[t]{3}{*}{0,33} \\
\hline Regular & 172 & 62 & 35,3 & $1,44(0,85-2,43)$ & \\
\hline Ruim/muito ruim & 40 & 15 & 38,7 & $1,67(0,73-3,84)$ & \\
\hline \multicolumn{6}{|l|}{ Dor $>24$ meses } \\
\hline Sim & 187 & 66 & 34,4 & $1,04(0,59-1,84)$ & \multirow[t]{2}{*}{0,87} \\
\hline Não & 113 & 38 & 33,4 & 1,00 & \\
\hline \multicolumn{6}{|l|}{ Dor pelve/pernas } \\
\hline Sim & 103 & 42 & 39,1 & $1,44(0,92-2,24)$ & \multirow[t]{2}{*}{0,10} \\
\hline Não & 200 & 62 & 30,9 & 1,00 & \\
\hline \multicolumn{6}{|c|}{ Dor moderada/forte/intensa } \\
\hline Sim & 239 & 89 & 36,0 & $1,53(0,80-2,93)$ & \multirow[t]{2}{*}{0,19} \\
\hline Não & 54 & 14 & 26,8 & 1,00 & \\
\hline \multicolumn{6}{|l|}{ Dor todos os dias } \\
\hline Sim & 146 & 52 & 35,2 & $1,18(0,71-1,98)$ & \multirow[t]{2}{*}{0,50} \\
\hline Não & 153 & 50 & 31,4 & 1,00 & \\
\hline
\end{tabular}

* Calculado com base nos pesos amostrais decorrentes do desenho amostral complexo. OR, Odds Ratio. 
Tabela 3. Relação entre medicamentos antirreumáticos e anti-inflamatórios e características de uso e acesso aos serviços de saúde, autopercepção de saúde e características da dor que mais incomoda entre os idosos com dor crônica (n=303) - Estudo SABE, 2010, São Paulo.

\begin{tabular}{|c|c|c|c|c|c|}
\hline Variáveis & Total* & $\mathbf{n}$ & $\%$ & OR não ajustado (IC95\%) & $\mathbf{p}$ \\
\hline \multicolumn{6}{|l|}{ Plano de saúde } \\
\hline Sim & 118 & 16 & 13,3 & 1,00 & \multirow[t]{2}{*}{0,86} \\
\hline Não & 185 & 24 & 12,5 & $0,93(0,44-1,97)$ & \\
\hline \multicolumn{6}{|c|}{ Dificuldade para acessar/utilizar serviços de saúde } \\
\hline Sim & 112 & 14 & 11,3 & $0,80(0,38-1,69)$ & \multirow[t]{2}{*}{0,56} \\
\hline Não & 190 & 26 & 13,7 & 1,00 & \\
\hline \multicolumn{6}{|c|}{ Financiamento da última consulta médica } \\
\hline SUS & 164 & 23 & 12,9 & 1,00 & \multirow[t]{2}{*}{0,90} \\
\hline Convênio/Particular & 107 & 14 & 13,5 & $1,05(0,48-2,29)$ & \\
\hline \multicolumn{6}{|l|}{ Percepção de saúde } \\
\hline Muito boa/boa & 79 & 8 & 10,6 & 1,00 & \multirow[t]{3}{*}{1,00} \\
\hline Regular & 172 & 25 & 13,8 & $1,35(0,48-3,78)$ & \\
\hline Ruim/muito ruim & 40 & 6 & 14,1 & $1,38(0,47-4,00)$ & \\
\hline \multicolumn{6}{|l|}{ Dor $>24$ meses } \\
\hline Sim & 187 & 27 & 13,9 & $1,25(0,60-2,60)$ & \multirow[t]{2}{*}{0,53} \\
\hline Não & 113 & 13 & 11,4 & 1,00 & \\
\hline \multicolumn{6}{|l|}{ Dor pelve/pernas } \\
\hline Sim & 103 & 14 & 12,8 & $1,00(0,44-(2,24)$ & \multirow[t]{2}{*}{0,99} \\
\hline Não & 200 & 26 & 12,8 & 1,00 & \\
\hline \multicolumn{6}{|c|}{ Dor moderada/forte/intensa } \\
\hline Sim & 239 & 37 & 14,8 & $2,63(0,70-9,94)$ & \multirow[t]{2}{*}{0,14} \\
\hline Não & 54 & 3 & 6,2 & 1,00 & \\
\hline \multicolumn{6}{|l|}{ Dor todos os dias } \\
\hline $\operatorname{Sim}$ & 146 & 23 & 16,4 & $1,77(0,79-3,96)$ & \multirow[t]{2}{*}{0,16} \\
\hline Não & 153 & 17 & 10,0 & 1,00 & \\
\hline
\end{tabular}

* Calculado com base nos pesos amostrais decorrentes do desenho amostral complexo. OR, Odds Ratio.

Tabela 4. Relação entre medicamentos antidepressivos e características de uso e acesso aos serviços de saúde, autopercepção de saúde e características da dor que mais incomoda entre os idosos com dor crônica $(n=303)$ - Estudo SABE, 2010, São Paulo.

\begin{tabular}{|c|c|c|c|c|c|}
\hline Variáveis & Total* & $\mathbf{n}$ & $\%$ & OR não ajustado (IC95\%) & $\mathbf{p}$ \\
\hline \multicolumn{6}{|l|}{ Plano de saúde } \\
\hline Sim & 118 & 21 & 17,6 & 1,00 & 0,04 \\
\hline Não & 185 & 18 & 9,5 & $0,49(0,24-0,98)$ & \\
\hline \multicolumn{6}{|c|}{ Dificuldade para acessar/utilizar serviços de saúde } \\
\hline Sim & 112 & 17 & 15,3 & $1,50(0,74-3,07)$ & 0,25 \\
\hline Não & 190 & 21 & 10,7 & 1,00 & \\
\hline \multicolumn{6}{|c|}{ Financiamento da última consulta médica } \\
\hline SUS & 164 & 20 & 12,5 & 1,00 & 0,61 \\
\hline Convênio/Particular & 107 & 17 & 14,5 & $1,18(0,60-2,31)$ & \\
\hline \multicolumn{6}{|l|}{ Percepção de saúde } \\
\hline Muito boa/boa & 79 & 13 & 16,0 & 1,00 & 0,21 \\
\hline Regular & 172 & 16 & 9,1 & $0,52(0,24-1,15)$ & \\
\hline Ruim/muito ruim & 40 & 8 & 19,1 & $1,24(0,44-3,48)$ & \\
\hline \multicolumn{6}{|l|}{ Dor $>24$ meses } \\
\hline Sim & 187 & 27 & 13,7 & $1,28(0,60-2,75)$ & 0,51 \\
\hline Não & 113 & 12 & 11,0 & 1,00 & \\
\hline \multicolumn{6}{|l|}{ Dor pelve/pernas } \\
\hline Sim & 103 & 14 & 12,7 & $1,01(0,51-2,01)$ & 0,97 \\
\hline Não & 200 & 25 & 12,5 & 1,00 & \\
\hline \multicolumn{6}{|c|}{ Dor moderada/forte/intensa } \\
\hline Sim & 239 & 38 & 15,7 & & \\
\hline Não & 54 & - & - & 1,00 & \\
\hline \multicolumn{6}{|l|}{ Dor todos os dias } \\
\hline Sim & 146 & 21 & 15,1 & $1,48(0,76-2,89)$ & 0,24 \\
\hline Não & 153 & 18 & 44,9 & 1,00 & \\
\hline
\end{tabular}


Tabela 5. Relação entre medicamentos sintomáticos para dispepsia e características de uso e acesso aos serviços de saúde, autopercepção de saúde e características da dor que mais incomoda entre idosos com dor crônica (n-303) - Estudo SABE, 2010, São Paulo.

\begin{tabular}{|c|c|c|c|c|c|}
\hline Variáveis & Total* & $\mathbf{n}$ & $\%$ & OR não ajustado (IC95\%) & $\mathbf{p}$ \\
\hline \multicolumn{6}{|l|}{ Plano de saúde } \\
\hline Sim & 118 & 38 & 30,8 & 1,00 & \multirow[t]{2}{*}{0,44} \\
\hline Não & 185 & 52 & 26,8 & $0,82(0,49-1,36)$ & \\
\hline \multicolumn{6}{|c|}{ Dificuldade para acessar/utilizar serviços de saúde } \\
\hline Sim & 112 & 42 & 34,6 & $1,63(0,89-2,97)$ & \multirow[t]{2}{*}{0,10} \\
\hline Não & 190 & 47 & 24,5 & 1,00 & \\
\hline \multicolumn{6}{|c|}{ Financiamento da última consulta médica } \\
\hline SUS & 164 & 50 & 30,2 & 1,00 & \multirow[t]{2}{*}{0,85} \\
\hline Convênio/Particular & 107 & 35 & 29,3 & $1,04(0,63-1,72)$ & \\
\hline \multicolumn{6}{|l|}{ Percepção de saúde } \\
\hline Muito boa/boa & 79 & 14 & 18,2 & 1,00 & \multirow[t]{3}{*}{0,04} \\
\hline Regular & 172 & 59 & 32,9 & $2,20(1,12-4,32)$ & \\
\hline Ruim/muito ruim & 40 & 16 & 34,1 & $2,33(0,90-5,97)$ & \\
\hline \multicolumn{6}{|l|}{ Dor $>24$ meses } \\
\hline Sim & 187 & 59 & 30,1 & $1,20(0,68-2,11)$ & \multirow[t]{2}{*}{0,50} \\
\hline Não & 113 & 31 & 26,3 & 1,00 & \\
\hline \multicolumn{6}{|l|}{ Dor pelve/pernas } \\
\hline Sim & 103 & 37 & 33,1 & $1,41(0,83-2,39)$ & \multirow[t]{2}{*}{0,20} \\
\hline Não & 200 & 53 & 26,0 & 1,00 & \\
\hline \multicolumn{6}{|c|}{ Dor moderada/forte/intensa } \\
\hline Sim & 239 & 75 & 30,0 & $1,41(0,61-3,25)$ & \multirow[t]{2}{*}{0,41} \\
\hline Não & 54 & 14 & 23,3 & 1,00 & \\
\hline \multicolumn{6}{|l|}{ Dor todos os dias } \\
\hline Sim & 146 & 58 & 37,1 & $2,24(1,31-3,81)$ & \multirow[t]{2}{*}{0,003} \\
\hline Não & 153 & 31 & 20,9 & 1,00 & \\
\hline
\end{tabular}

* Calculado com base nos pesos amostrais decorrentes do desenho amostral complexo. OR, Odds Ratio.

\section{DISCUSSÃO}

Os resultados deste estudo demonstram uma população de idosos com predominância do sexo feminino, a maioria com até 79 anos, casada e com baixa escolaridade. Mais da metade considera sua renda suficiente para o sustento; tem perfil de doenças crônico degenerativas do sistema cardiovascular e osteomuscular; maior frequência de utilização do SUS e alta frequência de realização de consultas médicas nos últimos seis meses.

Os dados sociodemográficos deste estudo apresentam em sua maioria semelhança com os demais estudos realizados no Brasil. Houve similaridade da quantidade de mulheres e idosos casados na população pesquisada com um estudo realizado com idosos em um município do estado de Santa Catarina [13].

Da mesma forma, o estudo realizado com idosos no município de Cuiabá, Mato Grosso, apresentou como semelhança a predominância do sexo feminino $61,6 \%$; estado civil casado em $54,7 \%$ e escolaridade até 4 anos, 43,0\% [14]. Essas características representam as condições do envelhecimento populacional de diversos países inclusive o Brasil.
O perfil de morbimortalidade com relatos de hipertensão, diabetes e doenças osteoarticulares coincidem com outros estudos, com variações das frequências $[14,15]$. A hipertensão mostrou-se uma das principais comorbidades em diversos estudos realizados em diferentes locais do Brasil, sendo que as frequências variaram de $32,2 \%$ a $72 \%[14,15]$.

O uso e o acesso aos serviços de saúde são fatores importantes para a qualidade da saúde dos idosos. Em $2010,46,8 \%$ dos idosos relataram possuir plano de saúde privado, o que significou um aumento de 5,4\% de adesão aos planos de saúde privados se comparados aos dados do Estudo SABE do ano de 2000, já que nesse ano, $41,4 \%$ dos idosos relataram possuir planos de saúde privados [16].

Em relação à dificuldade para acessar e utilizar serviços de saúde $24,4 \%$, dos idosos da pesquisa mencionou ter dificuldades, todavia, $81 \%$ teve sua consulta médica há menos de seis meses e, a principal fonte de financiamento da última consulta médica foi o SUS, representando 46,6\%. Esta mesma perspectiva, avaliada nacionalmente, foi objeto de estudo da rede internacional de estudos sobre envelhecimento Saúde dos Idosos Brasileiros, evidenciando que $75,3 \%$ dos 
idosos necessitam exclusivamente do SUS [17]. Sendo assim, os dois estudos mostram acentuada dependência dos idosos em relação a assistência à saúde pública brasileira.

O resultado dessa pesquisa demonstra que apenas $32,6 \%$ dos idosos não gastaram com medicações. O restante gastou em média 134,21 reais ou 79,64 dólares. Em 2010, o salário mínimo era de 510,00 reais, o que equivaleria a cerca de 310,00 dólares, correspondendo o gasto com medicações cerca de $25,8 \%$ do salário mínimo da época. Atualmente, com o salário mínimo em 937,00 reais ou 284,00 dólares, o gasto seria de 241,74 reais ou 73,25 dólares [18-21]. Dessa maneira, os gastos com medicações pelos idosos comprometeram aproximadamente a um quarto da renda daqueles que receberam um salário mínimo, impactando significativamente na renda dos mesmos.

A prevalência de dor crônica encontrada foi de $30,98 \%$. Para a dor que mais incomoda, observou-se frequência diária, de intensidade forte a moderada e de longa duração. Essa prevalência é inferior ao estudo realizado com idosos residentes na área de abrangência de uma unidade básica de saúde, localizada na região norte da cidade de Londrina. Tal diferença pode ter ocorrido porque no estudo de Londrina os idosos possuíam vínculo com o serviço de saúde [22]. Porém, é similar a outros estudos realizados com idosos do SABE [11].

A dor crônica influencia negativamente a qualidade de vida dos idosos, no domínio físico e nas relações sociais. Um estudo com 934 idosos de Goiânia observou-se que a dor se associou à fadiga, aos distúrbios do sono, à dependência de tratamentos e ao uso de medicamentos [23].

Os analgésicos possuem um importante papel para o estabelecimento de uma terapêutica adequada do quadro doloroso. Porém, os idosos são mais suscetíveis de apresentarem manifestações colaterais das drogas do que os indivíduos jovens, em virtude das alterações funcionais próprias do processo de envelhecimento [24].

A escolha do tratamento medicamentoso esbarra ainda em outros aspectos, pois cada droga tem farmacocinética e farmacodinâmica própria, influenciando a homeostase e o estado de saúde do usuário. Acrescenta-se que os idosos fazem, normalmente, uso de várias medicações em decorrência de problemas crônicos ou agudos, o que os torna mais passíveis a interações medicamentosas prejudiciais [25].

Em um estudo realizado com população residente em lares de idosos na Austrália, quase (91\%) dos residentes receberam analgésicos prescritos. Destes,
$2.057(28,1 \%)$ estavam tomando opioides regulares e apenas $50 \%$ daqueles que tomaram opioides regularmente receberam paracetamol em doses de 3-4 g/dia. Dentre àqueles que tomaram opioides regulares e algum ansiolítico/hipnótico o número foi de $48(48,4 \%)$. Isso mostra uma grande diferença nos resultados do presente estudo, na qual apenas $26,7 \%$ referiu utilizar analgésicos. E das outras drogas usadas como adjuvantes ou isoladamente para o tratamento da dor nos idosos, o resultado também foi abaixo comparado ao estudo australiano. Esta diferença pode ser explicada pelo fato de o estudo australiano ter como população de estudo idosos institucionalizados [24].

Em outro estudo realizado em uma unidade de saúde em município do interior do Paraná, do total de idosos com dor crônica $(80,4 \%)$ referiram fazer uso de medicamentos analgésicos. Citaram em primeiro lugar os analgésicos simples (54\%), principalmente dipirona e paracetamol, seguidos de anti-inflamatórios $(38,2 \%)$, miorrelaxantes $(9,3 \%)$, espamolíticos $(3,7 \%)$, tranquilizantes menores $(2,8 \%)$ e antidepressivos (1,9\%). Quando comparados os resultados destes estudos, o uso de analgésicos e anti-inflamatórios do nosso estudo foram menores, já as outras medicações tiveram resultados similares [22]. Neste caso, apesar das semelhanças de resultado, a população de pesquisa vinculada aos serviços de saúde pode ter mais comorbidades e estar mais medicalizada do que a população de idosos da comunidade não selecionados a partir do vínculo com serviços de saúde.

Entretanto, no presente estudo $46,0 \%$ dos idosos referiram dor de frequência diária, com intensidade forte a moderada $(40,0 \%)$ e de longa duração $(62,3 \%)$ o que pode caracterizar um sub tratamento. Mesmo entre os idosos que apresentaram dor moderada a intensa, houve baixa frequência de uso de medicações analgésicas e de opioides, reforçando a hipótese de subtratamento.

A prescrição das medicações antirreumáticas e antiinflamatórias, psicolépticos, depressores do sistema nervoso, e psicoanalépticos, que estimulam a atividade cerebral, não foi influenciada pelas características de uso e acesso aos serviços de saúde, percepção de saúde e características da dor. Isso pode ter ocorrido devido ao número pequeno de indivíduos usuários destes medicamentos na população pesquisada. Porém, o uso de anti-inflamatórios por idosos foi citado em um estudo sobre polifarmácia do estudo SABE de 2006 como um dos medicamentos mais usados no tratamento da dor e relacionado a polifarmácia [26].

Os psicolépticos e psicoanalépticos, também conhecidos como drogas sedativas e psicotrópicas, são 
usadas como drogas adjuvantes, ou isoladamente para o tratamento de dor, ou ainda para outras finalidades terapêuticas [8]. Embora sejam medicações indicadas para condições diversas, neste estudo observou-se baixo número de idosos que referiram seu uso.

Estudo realizado em idosos frágeis institucionalizados em Juiz de Fora, Minas Gerais, mostrou que $90,2 \%$ usava medicamentos com ação no sistema nervoso, e dos medicamentos potencialmente inapropriados segundo a pesquisa, os psicolépticos, como: diazepan, tioridazina e flurazepan e os psicoanalépticos, fluoxetina e amitriptilina apareceram como de alto risco. Os profissionais de saúde precisam estar atentos ao uso inadequado dessas medicações em idosos [27].

O uso de antiácidos esteve relacionado à autopercepção regular da saúde e ao relato de dor diária. O uso dos antiácidos por idosos necessita ser muito bem avaliado, pois podem ser usados para uma variedade de indicações [28].

Por alterar o $\mathrm{pH}$ gástrico, tais medicamentos interferem na absorção de outras drogas e nutrientes, como o cálcio, o que pode aumentar o risco de osteoporose [28]. Além disso, o seu uso associado a analgésicos e anti-inflamatórios precisa ser adequadamente avaliado pelos prescritores.

O conceito de dor atual envolve aspectos psicológicos (ansiedade e depressão); sociais (crenças, papéis sociais e relacionamentos) e espirituais, (sofrimento e significado da dor). Assim, limitar o controle da dor somente às intervenções medicamentosas parece hoje completamente inadequado. A dor nos idosos também deve ser tratada com medidas não farmacológicas como: terapia de aquecimento superficial, terapia de resfriamento superficial, massagem, acupuntura, acupressão, estimulação elétrica nervosa transcutânea, exercícios regulares, biofeedback, hipnose e autohipnose, meditação, estratégias de enfrentamento, que apresentam grande eficácia e menos efeitos colaterais que o tratamento farmacológico. Conforme avaliação podem ser usadas associadas com medicamentos ou isoladamente [8].

Em estudo sobre qualidade do sono em idosos com dores crônicas, residentes de um município catarinense, o que mais se destacou foi que quanto maior o número de doenças crônicas autorrelatadas, pior era a qualidade do sono. Neste cenário, tem-se que o número elevado de doenças crônicas aumenta as chances de interações medicamentosas, em meio a um ciclo que pode gerar diferentes tipos de sofrimento físico e psíquico. Isso revela que eles vivem o dia-adia sofrendo e impedidos de realizar atividades, de interagir com outras pessoas e até mesmo de manter o padrão do sono adequado [29].

Os idosos sofrem com a dor por muito tempo, pois quanto à dor que mais incomoda, a maioria $(62,3 \%)$, referiu dor há mais de 24 meses e $24,4 \%$ mencionaram dificuldade em acessar/utilizar serviços de saúde.

Dos idosos com dor crônica que referiram dor moderada/forte/intensa, somente $47,7 \%$ faziam uso de medicações para dor, o que pode indicar o pouco uso de medicamento sintomático da dor na prescrição dos profissionais.

Dados sobre uso de terapêuticas analgésicas não medicamentosas não foram avaliados. Porém, a baixa frequência de medicamentos para controle da dor sugere que os idosos se utilizam de estratégias não medicamentosas para o enfrentamento da dor, permitindo assim que mesmo aqueles com dores consideradas crônicas e intensas não usem somente medicamentos para seu controle. A autonomia e o aprendizado pelo idoso de métodos de enfrentamento e controle da dor, além dos farmacológicos, precisam ser avaliados pelos profissionais de saúde, visando garantir a manutenção da capacidade funcional e qualidade de vida desta população.

Nos idosos com dor crônica, a consulta médica por convênio/particular associou-se a menor frequência de uso de analgésicos, resultado que pode estar relacionado ao maior acesso às práticas alternativas para o tratamento da dor como fisioterapia, acupuntura, entre outras. Por outro lado, idosos sem plano de saúde apresentaram menor frequência de uso de antidepressivos, quando comparados aos idosos com plano de saúde. Estes resultados alertam para as diferenças de conduta terapêutica entre serviços pagos e os públicos e chamam a atenção para a necessidade de maior capacitação dos profissionais de saúde.

O grupo de idosos com dor crônica com autopercepção de saúde regular e os que relataram dor todos os dias referiram mais frequentemente utilizar medicamentos sintomáticos para dispepsia, o que pode se mostrar como casos de uso da medicação indiscriminadamente, sem avaliação correta do profissional ou de automedicação.

O presente estudo apresenta limitações. A amostra analisada corresponde aos sobreviventes da coorte de 2000, reentrevistados em 2010, com maior frequência de mulheres. Segundo estudos internacionais e nacionais, as mulheres apresentam maior prevalência de dor. Além disso, não foi possível verificar se algum medicamento foi utilizado exclusivamente para o controle da dor crônica. 
A dor é um fator que afeta diretamente a vida do idoso e, estes muitas vezes, buscam o alívio para seu sofrimento nos medicamentos. Os profissionais de saúde, cuidadores e familiares devem considerar o idoso na sua singularidade, de forma que seja realizado o tratamento medicamentoso e não medicamentoso mais adequado, diminuindo os riscos da polifarmácia e amenizando a dor.

O presente estudo evidenciou que, entre os idosos que convivem com a dor crônica, ter percepção de saúde como regular, dor de frequência diária, realizar consultas médicas financiadas por serviço público e ter plano de saúde aumentam a frequência de utilização de determinados medicamentos, quando comparados com os demais idosos em estudo.

\section{NOTAS}

Parte deste trabalho é resultado de dissertação de mestrado de um dos autores (EGA), intitulada "Fatores associados ao uso de medicamentos para controle da dor por idosos: estudo SABE".

\section{Apoio financeiro}

Este estudo foi financiado pela Organização Pan-Americana de Saúde no ano de 2000 e pelo Governo do Estado de São Paulo a partir de 2006.

\section{Declaração de conflito de interesses}

Os autores declaram não haver conflitos de interesses relevantes ao conteúdo deste estudo.

\section{Contribuições dos autores}

Todos os autores fizeram contribuições substanciais para concepção, ou delineamento, ou aquisição, ou análise ou interpretação de dados; e redação do trabalho ou revisão crítica; e aprovação final da versão para publicação.

\section{Disponibilidade dos dados e responsabilidade pelos resultados}

Todos os autores declaram ter tido total acesso aos dados obtidos e assumem completa responsabilidade pela integridade destes resultados.

\section{REFERÊNCIAS}

1. Organização das Nações Unidas. População mundial deve ter mais 2 bilhões de pessoas nos próximos 30 anos [internet]. Nova York: ONU; 2019 - [updated 2019 June 17; cited 2019 Aug 20]. Available from: https://news.un.org/ pt/story/2019/06/1676601

2. Brasil. Portal Brasil. Em 10 anos, cresce número de idosos no Brasil [internet]. Brasília; 2016 - [cited 2018 May 14 ]. Available from: http:/www.brasil.gov.br/economia-e-emprego/2016/12/em-10-anos-cresce-numero-de-idosos-no-brasil. https://doi.org/10.14393/ufu.di.2019.1244

3. Coelho MAGM, Penha DSG, Mitre NCD, Lopes RA. Perfil de idosos do município de Itaúna/MG e influência da atividade física na dor crônica e na capacidade funcional. Fisioter. Bras. 2017;12(2);94-9. https://doi.org/10.33233/ fb.v12i2.806

4. Nicholas M, Vlaeyen JWS, Riefe W, Barkee A, Azizf Q, Benolielg R, Cohenh M, Eversi S, Giamberardinoj MA, Goebelk A, Korwisie B, Perrotl S, Svenssonm P, Wango SJ, Treede RD. The IASP classification of chronic pain for ICD-11: chronic primary pain. 2019;160:28-37. Available from: https://journals.lww.com/pain/Fulltext/2019/01000/ The_IASP_classification_of_chronic_pain_for.4.aspx. https://doi.org/10.1097/j.pain.0000000000001390

5. Dellaroza MSG, Pimenta CAM, Duarte YA; Lebrão ML. Dor crônica em idosos residentes em São Paulo, Brasil: prevalência, características e associação com capacidade funcional e mobilidade. Cad. Saúde Pública 2013;29(2): 325-334. http://dx.doi.org/10.1590/S0102-311X2013000200019

6. Cunha LL, Mayrin KWC. Influência da dor crônica na qualidade de vida em idosos. Rev. Dor. 2011;12(2):120-24. http://dx.doi.org/10.1590/S1806-00132011000200008

7. Dos Anjos NKC, Filho SCHC, Miguel TS, Miguel BS, Miguel VS, Brito LC, Martins DT, Costa DA. Relato de caso de polifarmácia no idoso: até onde pode-se considerar iatrogenia. Braz. J. Surg. Clin. Res. 2017;19(2):96-99.

8. Figueiró JAB.; Consentino RCM. Dor crônica e envelhecimento. In: Carvalho Filho ET, Papaléo Netto M. Geratria: fundamentos, clínica e terapêutica. 2a ed. São Paulo: Atheneu; 2005:677-684.

9. Sales AS, Sales MGS, Casotti, CA. Perfil farmacoterapêutico e fatores associados à polifarmácia entre idosos de Aiquara, Bahia, em 2014. Epidemiol. Serv. Saúde. 2017; 26(1):121-32. http://dx.doi.org/10.5123/s167949742017000100013

10. Palloni A, Peláez M. Histórico e natureza do estudo. In: Lebrão ML, Duarte YAO. SABE: saúde, bem-estar e envelhecimento: o projeto sabe no município de São Paulo: uma abordagem inicial. Brasília: Organização Pan-Americana da Saúde; 2003. p. 15-32. https://doi.org/10.11606/d.6.2007.tde-05122007-083756

11. Faculdade de Saúde Pública Universidade de São Paulo. Departamento de Epidemiologia. SABE. Saúde, Bem Estar e Envelhecimento. Estudo longitudinal de múltiplas coortes sobre as condições de vida e saúde dos idosos do Município de São Paulo [internet]. São Paulo; USP - FSP; 2015 - [updated 2015 Oct; cited 2019 May 14] Available 
from: https://bv.fapesp.br/pt/auxilios/89845/estudo-sabe-estudo-longitudinal-de-multiplas-coortes-sobre-as-condicoes-devida-e-saude-dos-idosos/. https://doi.org/10.11606/t.6.2011.tde-20122011-114524

12. Organização Mundial da Saúde. Guidelines for ATC classification and DDD assignment 2019 [internet]. Oslo, Norway: OMS; 2018 - [updated 2019 June 17; cited 2019 Aug 21]. Available from: https://www.whocc.no/filearchive/ publications/2019_guidelines_web.pdf

13. Gavasso WC, Beltrame V. Capacidade funcional e morbidades referidas: uma análise comparativa em idosos. Rev. Bras. Geriatr. Gerontol. 2017;20(3):399-409. http://dx.doi.org/10.1590/1981-22562017020.160080

14. Sudré MRS, Reiners AAO, Azevedo RCS, Floriano LA. Características socioeconômicas e de saúde de idosos assistidos pelas equipes de saúde a família. Ciênc., Cuid. Saúde, 2014:14(1):933-40. https://doi.org/10.4025/ cienccuidsaude.v14i1.19794

15. Tavares DMS, DIAS FA. Capacidade funcional, morbidades e qualidade de vida de idosos. Texto \& Contexto Enfermagem, Florianópolis. 2012;21(1):112-20. http://dx.doi.org/10.1590/S0104-07072012000100013

16. Louvison MCP, Lebrão MC, Duarte YAO, Santos JLF, Malik AM, Almeida ES. Desigualdades no uso e acesso aos serviços de saúde entre idosos do município de São Paulo. Rev. Saúde Públ. 2008;42(4):733-40. http://dx.doi.org/ $10.1590 /$ S0034-89102008000400021

17. Sociedade Brasileira de Geriatria e Gerontologia. OMS divulga metas para 2019: desafios impactam a vida de idosos [internet]. Rio de Janeiro:SBGG; 2019 - [updated 2019 Jan 27; cited 2019 Aug 20] Available from: https://sbgg.org.br/oms-divulga-metas-para-2019-desafios-impactam-a-vida-de-idosos/. https://doi.org/10.35667/ metasenf.2019.22.1003081360

18. Brasil. Lei no 12.255 , de 15 de junho de 2010. Dispõe sobre o salário mínimo a partir de 1 o de janeiro de 2010 , estabelece diretrizes para a política de valorização do salário mínimo entre 2012 e 2023 e revoga a Lei no 11.944, de 28 de maio de 2009. Diário Oficial [da] República Federativa do Brasil [internet]. [cited 2019 May 14]. Available from: http://www.planalto.gov.br/ccivil_03/_ato2007-2010/2010/lei/112255.htm. https://doi.org/10.11606/d.8.2017.tde26052017-125934

19. Brasil. Decreto no 8.948, de 29 de dezembro de 2016. Regulamenta a Lei no 13.152 , de 29 de julho de 2015 , que dispõe sobre o valor do salário mínimo e a sua política de valorização de longo prazo Diário Oficial [da] República Federativa do Brasil. [internet]. 2016 [cited 2019 May 14]. Available from: http:/www.planalto.gov.br/ccivil_03/ ato2015-2018/2016/decreto/D8948.htm. https://doi.org/10.15628/rbep.2009.2954

20. Brasil. Banco Central do Brasil. Dólar, euro e risco país: cotações diárias para o mês de dezembro/2010 [Internet]. Brasília: BCB; 2010 [cited 2019 May 14]. Available from: http://www.portalbrasil.net/2010/economia/dolar_riscopais dezembro.htm

21. Brasil. Banco Central do Brasil. Dólar, euro e risco país: cotações diárias para o mês de novembro / 2017 [Internet]. Brasília: BCB; 2017 - [cited 2019 May 14]. Available from: http://www.portalbrasil.net/2017/indices/dolar_riscopais_ novembro.htm

22. Dellaroza MSG, Furuya RK, Cabrera MAS, Matsuo T, Trelha C, Yamada KN, Pachola L. Caracterização da dor crônica e métodos analgésicos utilizados por idosos da comunidade. Rev Assoc Med Bras. 2008;54(1):36-41. https://doi.org/10.1590/s0104-42302008000100018

23. Santos TRA, Lima DM, Nakatani AYK, Pereira LV, Leal GS, Amaral RG. Consumo de medicamentos por idosos. Rev Saúde Pública. 2013;47(1):94-103. http://dx.doi.org/10.1590/S0034-89102013000100013

24. Veal FC, Bereznicki LR, Thompson AJ, Peterson GM. Pharmacological management of pain in australian aged care facilities. Age and Ageing. 2014;43:851-56. https://doi.org/10.1093/ageing/afu072

25. Telles Filho PCP, Almeida AGP, Pinheiro MLP. Automedicação em idosos: um problema de Saúde Pública. Rev Enf UERJ. 2013;21(2):197-201.

26. Carvalho MFC, Romano-Lieber NS, Bergsten-Mendes G, Secoli SR, Ribeiro E, Lebrão ML, Duarte YAO. Polifarmácia entre idosos do Município de São Paulo: estudo SABE. Rev Bras. Epidemiol. 2012;15(4):817-27. http://dx.doi.org/10.1590/S1415-790X2012000400013

27. Fochat RC, Horsth RBO, Sette MS, Raposo NRB, Chicourel EL. Perfil de utilização de medicamentos por idosos frágeis institucionalizados na Zona da Mata Mineira, Brasil. Rev Ciênc Farm Bás e Apl. 2012;33(3):447-54.

28. Brunton LL, Hilal-Dandan R. Goodman \& Gilman: manual de farmacologia e terapêutica. 2a ed. Porto Alegre: $\mathrm{AMGH} ; 2015$.

29. Ferretti F, Santos DT, Giuriatti L, Gauer APM, Teo CRPA. Qualidade do sono em idosos com e sem dor crônica. BrJP. 2018;1(2):141-46. http://dx.doi.org/10.5935/2595-0118.20180027 C 